\title{
THE TROTTER PRODUCT FORMULA FOR PERTURBATIONS OF SEMIBOUNDED OPERATORS
}

BY WILLIAM G. FARIS

Communicated by F. Browder, September 28, 1966

1. Introduction. Let $A$ and $B$ be linear operators generating semigroups $\exp (t A)$ and $\exp (t B)$ in a Hilbert space. Then under suitable conditions,

$$
\exp (t(A+B))=\lim _{n \rightarrow \infty}(\exp ((t / n) A) \exp ((t / n) B))^{n} .
$$

This is the Trotter [6] product formula. Though it is a theorem in perturbation theory, it is related to the Feynman path in tegral representation of solutions of partial differential equations and provides the best mathematical realization of this idea presently known.

Feynman [1] considered the Schrödinger equation of quantum mechanics:

$$
i(d u(t) / d t)=-(1 / 2 m) \Delta u(t)+V u(t)
$$

for $u(t)$ in $L^{2}\left(R^{3}\right)$, for each $t$, and with initial condition $u(0)=u$. Here $\Delta$ is the Laplace operator, and $V$ is a real valued function on $R^{3}$. The solution when $V=0$ is

$$
\begin{aligned}
u(x, t) & =(\exp ((i t / 2 m) \Delta) u)(x) \\
& =(2 \pi i t / m)^{-3 / 2} \int \exp \left[i \frac{1}{2} m\left(|x-y|^{2} / t\right)\right] u(y) d y .
\end{aligned}
$$

If we take $A=(i / 2 m) \Delta$ and $B=-i V$, then as Nelson [4] observed, the Trotter formula gives

$$
\begin{aligned}
u(x, t)= & (\exp ((i t / 2 m) \Delta-i t V) u)(x) \\
= & \lim _{n \rightarrow \infty}(\exp ((i t / 2 m n) \Delta) \exp (-(i t / n) V))^{n} u(x) \\
= & \lim _{n \rightarrow \infty} \int \cdots \int \exp \left[i \sum _ { j = 1 } ^ { n } \left\{\frac{1}{2} m \frac{\left|x_{j}-x_{j-1}\right|^{2}}{(t / n)^{2}}\right.\right. \\
& \left.\left.-V\left(x_{j-1}\right)\right\} \frac{t}{n}\right] u\left(x_{0}\right)(2 \pi i t / n m)^{-3 n / 2} d x_{0} \cdots d x_{n-1},
\end{aligned}
$$

where $x_{n}=x$, as a representation of the solution of the full Schrödinger equation. 
This is formally an integral over the space of all paths $x(\tau), 0 \leqq \tau$ $\leqq t$, in $R^{3}$ such that $x(t)=x$ of

$$
\exp \left[i \int_{0}^{t}\left\{\frac{1}{2} m(d x(\tau) / d \tau)^{2}-V(x(\tau))\right\} d \tau\right] u(x(0))
$$

which was Feynman's solution.

Here we state a version of the product formula for semigroups in Hilbert space. We consider a certain class of perturbations $B$ of $A$ so singular that the sum $A+B$ need not be densely defined when $A$ and $B$ are regarded as acting in the original Hilbert space. The formula is proved for abstract parabolic equations, and continuity in the mass parameter $m$ gives a definition of Feynman integral for the Schrödinger equation. Complete proofs of these results will appear elsewhere.

2. Perturbations of semibounded operators. Some constructions previously applied to partial differential equations by Lions [3] and Nelson [5] will be reviewed in this section.

If $K$ is a Hilbert space, the (conjugate) dual space $K^{*}$ is the Hilbert space of continuous antilinear functionals on $K$. We write $(v, u)$ for the value of $v$ in $K^{*}$ on $u$ in $K$.

An operator $A$ is a linear transformation from a linear subspace $D(A)$ of $K$ to $K^{*} . L\left(K, K^{*}\right)$ consists of those operators $A$ with $D(A)$ $=K$ and with $A$ continuous from $K$ to $K^{*}$.

Definition. A sector of the complex plane is a closed convex subset bounded by two rays meeting at 0 . Let $A$ be an operator and $S$ a sector. $A$ is said to be of type $S$ if $(A u, u) \in S$ for all $u$ in $D(A) . A$ is said to be dissipative if it is of type left half-plane.

Definition. Let $H$ be a Hilbert space with given inner product $(v, u)$. Use this inner product to identify $H$ with its (conjugate) dual space. Let $H^{1}$ be another Hilbert space with $H^{1} \subset H$ as a dense linear subspace such that the injection is continuous. (No distinguished norm in the family of equivalent norms on $H^{1}$ is specified.) Let $H^{-1}$ be the (conjugate) dual space of $H^{1}$. The dual of the given injection gives a continuous injection of $H$ into $H^{-1}$ which we may use to identify $H$ as a dense linear subspace of $H^{-1}$. The triple $H^{1} \subset H C H^{-1}$ constructed in this way is called a scale of Hilbert spaces.

If an operator $A$ in $H$ satisfies a suitable semiboundedness condition, it may be used to construct such spaces.

Proposition 1. Let $A$ be a densely defined operator in $H$ of type $S$, where $S-\{0\}$ is contained in the open left half-plane. Then there is a unique scale $H^{1} \subset H \subset H^{-1}$ such that 
(1) $D(A) \subset H^{1}$ and is dense in $H^{1}$.

(2) $A$ maps $D(A) \subset H^{1}$ continuously into $H^{-1}$, and hence extends by continuity to $\hat{A} \in L\left(H^{1}, H^{-1}\right)$.

(3) For each $\lambda>0$, an equivalent norm on $H^{1}$ may be given by $\|u\|_{1}^{2}$ $=\operatorname{Re}((-\hat{A}+\lambda) u, u)$.

If an operator $A_{0}$ generates a contraction semigroup $\exp t A_{0}$ on $H$, then for $u$ in $D\left(A_{0}\right), \operatorname{Re}\left(A_{0} u, u\right)=d\left(\left\|\exp \left(t A_{0}\right) u\right\|^{2}\right) / d t$, evaluated at $t=0$, and this is $\leqq 0$. So $A_{0}$ is dissipative.

Proposition 2. Let $H^{1} \subset H \subset H^{-1}$ be a scale. Let $A$ be a dissipative operator in $L\left(H^{1}, H^{-1}\right)$. Let $D\left(A_{0}\right)=\left\{u \in H^{1}: A u \in H\right\}$. Let $A_{0}$ be the restriction of $A$ to $D\left(A_{0}\right)$. Suppose that for some $\lambda>0$ and $c>0$,

$$
|((-A+\lambda) u, u)| \geqq c\|u\|_{1}^{2} .
$$

Then $A_{0}$ is the infinitesimal generator of a contraction semigroup $\exp \left(t A_{0}\right), t \geqq 0$, on $H$.

EXAMPLE. Let $H=L^{2}\left(R^{3}\right)$. If $V$ is a finite sum of real functions in various $L^{p}$ spaces, $2 \leqq p \leqq \infty$, then $-(1 / 2 m) \Delta+V$ is defined on $D(A)$ and is self-adjoint in $H$ (T. Kato [2]). Thus $(i / 2 m) \Delta-i V$ is the infinitesimal generator of a unitary group on $H$.

Since $D(\Delta)$ consists of continuous functions, if $V$ is not in $L^{2}$ near some point, then in general $-(1 / 2 m) \Delta+V$ is not self-adjoint in $H$. However, a perturbation theory based on the ideas of this section may sometimes be used to associate a self-adjoint operator in $H$ with these operators, which may be regarded as their sum.

Thus $\Delta$ is of type the negative real axis; so Proposition 1 applies. $H^{1}$ is the Sobolev space of functions in $L^{2}$ with first partial derivatives in $L^{2}$. If $V$ is a finite sum of real functions in various $L^{p}$ spaces, $3 / 2 \leqq p \leqq \infty$, then it can be shown that $V$ defines an operator in $L\left(H^{1}, H^{-1}\right)$. $(1 / 2 m) \Delta$ may also be regarded as an operator in $L\left(H^{1}, H^{-1}\right)$. So the sum $(i / 2 m) \Delta-i V$ is everywhere defined on $H^{1}$ and Proposition 2 may be used in the proof that it has a restriction which generates a unitary group on $H$.

3. The product formula. Theorem 1. Let $H$ be a Hilbert space. Let $A$ be the infinitesimal generator of a contraction semigroup in $H$. Assume that $A$ is of type $S$, where $S-\{0\}$ is contained in the open left half-plane. Let $H^{1} \subset H \subset H^{-1}$ be the scale associated with $A$. A extends by continuity to an operator $\hat{A}$ in $L\left(H^{1}, H^{-1}\right)$.

Let $B$ be the infinitesimal generator of a contraction semigroup in $H$. Asssme that 
(1) $D(B) \cap H^{1}$ is dense in $H^{1}$ and $B$ on this domain extends by continuity to an operator $\hat{B}$ in $L\left(H^{1}, H^{-1}\right)$.

(2) $(-B)^{1 / 2}$ and $\left(-B^{*}\right)^{1 / 2}$ may each be restricted to some domain dense in $H^{1}$ on which they are continuous from $H^{1}$ to $H$.

Then $\hat{A}+\hat{B}$ has a restriction $C$ which is the infinitesimal generator of a contraction semigroup in $H$. As $n \rightarrow \infty$ the products

$$
(\exp ((t / n) A) \exp ((t / n) B))^{n}
$$

converge to $\exp t C$ in the strong operator topology.

The proof of the first part of the result is immediate and illustrates the perturbation theory based on the ideas of the previous section. For since $\hat{A}$ and $\hat{B}$ are dissipative, $\hat{C}=\hat{A}+\hat{B} \in L\left(H^{1}, H^{-1}\right)$ is also. In addition,

$$
|((-\hat{C}+\lambda) u, u)| \geqq|\operatorname{Re}((-\hat{C}+\lambda) u, u)| \geqq \operatorname{Re}((-\hat{A}+\lambda) u, u)
$$

and the last term, as is stated in Proposition 1, dominates a positive multiple of $\|u\|_{1}^{2}$. Thus the hypotheses of Proposition 2 are satisfied, and $\hat{A}+\hat{B}$ has a restriction to $H$ which generates a contraction semigroup.

THEOREM 2. Let $A$ be the infinitesimal generator of a contraction semigroup in $H$. Assume that a complex number $\sigma$ with $|\sigma|=1$ may be chosen so that $\sigma A$ is of type $S$, where $S-\{0\}$ is contained in the open left half-plane. Let $H^{1} \subset H \subset H^{-1}$ be the scale associated with $\sigma A$. Let $\alpha$ be any complex number such that $\alpha A$ is dissipative.

Let $\hat{B}$ be a dissipative operator in $L\left(H^{1}, H^{-1}\right)$. Assume that some norm for $H^{1}$ of the form $\|u\|_{1}^{2}=\operatorname{Re}((-\sigma \hat{A}+k) u, u)$ may be chosen so that the operator norm of $\widehat{B}$ is then strictly less than $|\alpha|$.

Then $\alpha \hat{A}+\hat{B} \in L\left(H^{1}, H^{-1}\right)$ has a restriction to an operator $C_{\alpha}$ which generates a contraction semigroup $\exp \left(t C_{\alpha}\right) \cdot \exp \left(t C_{\alpha}\right)$ is strongly continuous in $\alpha$ for fixed $t$.

4. Application to the Schrödinger equation. Let the scale $H^{1} \subset H$ $C H^{-1}$ consist of the Sobolev spaces of the previous example. We assume $V$ to be a finite sum of real functions in various $L^{p}\left(R^{3}\right)$ spaces, $3 / 2 \leqq p \leqq \infty$. This implies that $V$ is bounded from $H^{1}$ to $H^{-1}$ with sufficiently small norm. In order to ensure that $V$ as an operator in $H$ has domain dense in $H^{1}$, we require that $V$ is locally in $L^{2}$ except on a closed set of capacity zero.

Then the above results may be used with $A=i \Delta, \alpha=1 /(2(m+i \epsilon))$, and $B=-i V . C_{\alpha}$ is obtained by restricting $\hat{C}_{\alpha}=\alpha \hat{A}+\hat{B}$, the sum of the corresponding operators in $L\left(H^{1}, H^{-1}\right)$. (The scale $H^{1} \subset H \subset H^{-1}$ 
is always that associated with $-i A=\Delta$.) When $\epsilon>0$, Theorem 1 applied to $\alpha A$ and $B$ gives

$$
\lim _{n \rightarrow \infty}(\exp ((t / n) \alpha A) \exp ((t / n) B))^{n}=\exp t C_{\alpha} .
$$

Then Theorem 2 shows that when $\epsilon \rightarrow 0+, \exp t C_{\alpha}$ converges to the same unitary group as was found in the example. We conclude that for $t \geqq 0$,

$$
\begin{aligned}
\lim _{c \rightarrow 0+} \lim _{n \rightarrow \infty} \int & \cdots \int \exp \left[i \sum _ { j = 1 } ^ { n } \left\{\frac{1}{2}(m+i \epsilon) \frac{\left|x_{j}-x_{j-1}\right|^{2}}{(t / n)^{2}}\right.\right. \\
& \left.\left.-V\left(x_{j-1}\right)\right\} \frac{t}{n}\right] u\left(x_{0}\right)(2 \pi i t / n(m+i \epsilon))^{-3 n / 2} d x_{0} \cdots d x_{n-1},
\end{aligned}
$$

where $x_{n}=x$, converges in $L^{2}$ to a function $u(x, t)$ which may be regarded as the solution of the Schrödinger equation with initial condition $u(x, 0)=u(x)$.

It is a pleasure to thank Professor Edward Nelson for his generous counsel.

\section{REFERENCES}

1. R. P. Feynman, Space-time approach to nonrelativistic quantum mechanics, Rev. Modern Phys. 20 (1948), 367-387.

2. T. Kato, Fundamental properties of Hamiltonian operators of Schrodinger type, Trans. Amer. Math. Soc. 70 (1951), 195-211.

3. J. L. Lions, Equations differentielles operationelles, Springer, Berlin, 1961.

4. E. Nelson, Feynman integral and the Schrödinger equation, J. Math. Phys. 5 (1964), 332-343.

5. E. Nelson, Operator differential equations, Notes for Mathematics, by John Cannon, Princeton University, Princeton, N. J., (1964-1965), p. 520.

6. H. F. Trotter, On the product of semigroups of operators, Proc. Amer. Math. Soc. 10 (1959), 545-551.

Cornell University 